\title{
Model multi-cloud parameterizations for convectively coupled waves: Detailed nonlinear wave evolution
}

\author{
Boualem Khouider $^{\mathrm{a}, *}$, Andrew J. Majda ${ }^{\mathrm{b}}$

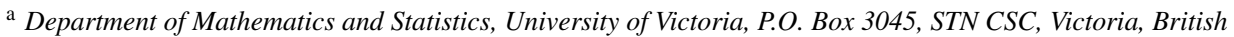 \\ Columbia V8W 3P4, Canada \\ ${ }^{\mathrm{b}}$ Department of Mathematics and Center for Atmosphere/Ocean Sciences, Courant Institute, \\ New York University, New York, NY, United States
}

Available online 18 September 2006

\begin{abstract}
Recent observational analysis reveals the central role of three cloud types, congestus, stratiform, and deep-convective cumulus clouds, in the dynamics of large scale convectively coupled Kelvin waves, westward propagating 2-day waves, and the Madden-Julian oscillation. Recently, a systematic model convective parametrization highlighting the dynamic role of the three cloud types has been developed by the authors involving two baroclinic modes of vertical structure: a deep-convective heating mode and a second mode with low level heating and cooling corresponding, respectively, to congestus and stratiform clouds. The model includes a systematic moisture equation where the lower troposphere moisture increases through detrainment of shallow cumulus clouds, evaporation of stratiform rain, and moisture convergence and decreases through deep-convective precipitation and also a nonlinear switch which favors either deep or congestus convection depending on the relative dryness of the middle troposphere. The detailed nonlinear evolution of large scale convectively coupled waves in the model parametrization is studied here in a chaotic intermittent regime of the nonlinear dynamics associated with weaker mean radiative cooling where such waves are isolated in space and time. This regime is utilized to elucidate in a clean fashion several novel features of the model parametrization. In particular, four stages of nonlinear wave evolution occur: in the preconditioning and birth stages, the role of congestus moistening and second baroclinic convergence are crucial while in the dying stage of the large scale convectively coupled wave, the role of the nonlinear switch, and the drying of the troposphere are essential. In the mature phase, the large scale features of the convectively coupled waves resemble those in observations of convectively coupled Kelvin waves including the propagation speed, wave tilt, temperature, heating, and velocity structure.
\end{abstract}

(C) 2006 Elsevier B.V. All rights reserved.

Keywords: Convectively coupled waves; Tropical convection; Convective parametrizations

* Corresponding author.

E-mail address: khouider@math.uvic.ca (B. Khouider). 


\section{Introduction}

Recent analysis of observations over the warm pool in the tropics reveals the ubiquity of three cloud types above the boundary layer: shallow congestus clouds, stratiform clouds, and deep penetrative cumulus clouds (Lin and Johnson, 1996; Johnson et al., 1999). Furthermore, recent analysis of convectively coupled waves on the large scales reveals a similar multi-cloud convective structure with leading shallow congestus cloud decks which moisten and precondition the lower troposphere followed by deep convection and finally trailing decks of stratiform precipitation; this structure applies to the eastward propagating convectively coupled Kelvin waves (Wheeler and Kiladis, 1999; Straub and Kiladis, 2002) and westward propagating 2day waves (Haertel and Kiladis, 2004) which reside on equatorial synoptic scales of order $1000-3000 \mathrm{~km}$ in the lower troposphere as well as the planetary scale Madden-Julian oscillation (Dunkerton and Crum, 1995; Kiladis et al., 2005). An inherently multi-scale theory for the Madden-Julian oscillation with qualitative agreement with observations which is based on these three cloud types has been developed recently (Majda and Biello, 2004; Biello and Majda, 2005).

Moreover, contemporary general circulation models (GCMs) often perform poorly in parametrizing and/or resolving the observed large scale features of organized tropical convection such as convectively coupled waves as well as their impact on planetary scale tropical circulation (Slingo et al., 1996; Moncrieff and Klinker, 1997); the reasons for such poor performance are not well understood. Simple models with crude vertical resolution, typically involving a single baroclinic vertical mode, have been used for theoretical and numerical studies of various strategies for parameterizing moist convection and convectively coupled waves (Emanuel, 1987; Mapes, 1993; Neelin and Yu, 1994; Yano et al., 1995, 1998; Majda and Shefter, 2001a; Majda and Khouider, 2002; Fuchs and Raymond, 2002; Frierson et al., 2004).

Model parameterizations with two convective heating modes systematically representing a deep-convective mode and stratiform mode have first appeared in the work of Mapes (2000). Majda and Shefter (2001b) (hereafter MS) proposed a much simpler systematic version of Mapes' model based on a Galerkin projection of the primitive equations onto the first two linear-baroclinic modes yielding a set of two shallow water systems. The first baroclinic system is heated by the deep-convective clouds while the second baroclinic system is heated aloft by the stratiform clouds. Direct numerical simulations carried out in Majda et al. (2004) (hereafter MKKSS) revealed the resemblance of many features of the moist gravity waves for the MS model and the real world convective superclusters as depicted in MKKSS and in observational papers (e.g. Straub and Kiladis, 2002). One visible shortcoming of the MS model is however its short-cutting of the role of the shallow/congestus heating as in the early Mapes' model. Also, inherited from the quasi-equilibrium school (Yano and Emanuel, 1991; Yano et al., 1998), the MS model uses very sensitive parameters which are nonphysically kept fixed/constant and spatially homogeneous, such as the precipitation efficiency and the area fraction of deep convection.

Recently, the authors proposed and analyzed a new model convective parametrization with all three cloud types, within a framework similar to the MS model (Khouider and Majda, 2006a). In addition to the deep-convective and stratiform clouds, the model carries cumulus congestus clouds which serve to heat the second baroclinic mode from below and cool it from above as in actual congestus cloud decks. The new model is based on a self-consistent derivation and it avoids many of the commonly used ad hoc 
parameters. ${ }^{1}$ One key feature is a systematically derived equation for the vertically integrated water vapor perturbation, from a mean vertical background moisture profile, forced by both the first baroclinic and second baroclinic (low-level) convergence within the physical constraints of conservation of vertically integrated moist static energy. Also the new model takes into account the dryness and moistness of the middle troposphere through a varying-inhomogeneous switch parameter, $\Lambda$, in order to shut off or favor deep convection and to increase or decrease the downdrafts from the cooling associated with evaporation of shallow clouds and stratiform rain. Moreover, the congestus convection is amplified whenever the middle troposphere is too dry to sustain deep convection and is shut off completely when deep convection is at its maximum. Furthermore, linear stability analysis (Khouider and Majda, 2006a) reveals stability at small scales and a new type of large scale instability in realistic parameter regimes despite the low level moisture convergence used in the parametrization. As shown in Khouider and Majda (2006a), this large scale instability mechanism is completely different from both the wind induced surface heat exchange (WISHE) instability (Emanuel, 1987; Emanuel et al., 1994; Neelin and Yu, 1994; Yano et al., 1998; Majda and Shefter, 2001a) and stratiform instability without WISHE (MS, MKKSS) as well as convective instability of the second kind (CISK) (Charney and Eliassen, 1964; Yamasaki, 1969; Hayashi, 1971; Lindzen, 1974). The new multi-cloud type parameterizations are to some extent a marriage between the two schools of thought leading to the low-level convergence and quasi-equilibrium (Arakawa and Shubert, 1974) models while retaining the three key cloud types in the observational record.

This paper describes the detailed nonlinear evolution of convectively coupled waves in the multi-cloud model parametrization. This is achieved by studying nonlinear solutions about a sounding associated with a radiative convective equilibrium (RCE) with a weak radiative cooling, $Q_{R, 1}^{0}=0.04 \mathrm{~K} \mathrm{day}^{-1}$ in contrast to the typical value $Q_{R, 1}^{0}=1 \mathrm{~K} \mathrm{day}^{-1}$. This regime slows down the radiative cooling in the troposphere (thus reducing the model's convective instability) while still possessing strongly nonlinear chaotic and intermittent dynamics involving appearance and demise of large scale convectively coupled gravity waves which are isolated in space and time. The various physical mechanisms in the multi-cloud model parametrization and their role in the birth and decay of the convectively coupled gravity waves are elucidated here. The study presented here is complementary to the one developed recently by the authors (Khouider and Majda, 2006b) with the standard stronger radiative cooling of $1 \mathrm{~K} \mathrm{day}^{-1}$ where the properties of the convectively coupled waves are inherently statistical in nature for idealized Walker cell simulations. The model parametrization is presented briefly in Section 2 while the nonlinear simulations are presented and analyzed in Section 3.

\section{The multi-cloud type model parameterizations}

Here, we summarize the model convective parametrization introduced recently by Khouider and Majda (2006a).

\subsection{The dynamical core}

The dynamical core of the model convective parametrization consists of two coupled shallow water systems involving a direct heating mode forced by a bulk precipitation rate from deep

\footnotetext{
${ }^{1}$ Certainly, many is not enough to "get rid" of all the ad hoc parameters one uses in a convective parametrization which is the price to pay for the crude vertical resolution. Several ad hoc parameters such as the moisture stratification $\tilde{Q}$ are still used here.
} 
penetrative clouds and a second baroclinic mode forced by both stratiform heating and congestus heating,

$$
\begin{aligned}
& \frac{\partial \mathbf{v}_{j}}{\partial t}+\overline{\mathbf{U}} \cdot \nabla \mathbf{v}_{j}+\beta y \mathbf{v}_{j}^{\perp}-\nabla \theta_{j}=-C_{\mathrm{d}}\left(u_{0}\right) \mathbf{v}_{j}-\frac{1}{\tau_{R}} \mathbf{v}_{j} \\
& \frac{\partial \theta_{1}}{\partial t}+\overline{\mathbf{U}} \cdot \nabla \theta_{1}-\operatorname{div} \mathbf{v}_{1}=\frac{\pi}{2 \sqrt{2}} P+S_{1} \\
& \frac{\partial \theta_{2}}{\partial t}+\overline{\mathbf{U}} \cdot \nabla \theta_{2}-\frac{1}{4} \operatorname{div} \mathbf{v}_{2}=\frac{\pi}{2 \sqrt{2}}\left(-H_{\mathrm{s}}+H_{\mathrm{c}}\right)+S_{2} .
\end{aligned}
$$

In (2.1), $\mathbf{v}_{j}=\left(u_{j}, v_{j}\right)_{j=1,2}$ represent the first and second baroclinic velocities assuming $G(z)=\sqrt{2} \cos \left(\frac{\pi z}{H_{\mathrm{T}}}\right)$ and $G(2 z)=\sqrt{2} \cos \left(\frac{2 \pi z}{H_{\mathrm{T}}}\right)$ vertical profiles, respectively, while $\theta_{j}, j=$ 1,2 are the corresponding potential temperature components with the vertical profiles $G^{\prime}(z)=$ $\sqrt{2} \sin \left(\frac{\pi z}{H_{\mathrm{T}}}\right)$ and $2 G^{\prime}(2 z)=2 \sqrt{2} \sin \left(\frac{2 \pi z}{H_{\mathrm{T}}}\right)$, respectively. Therefore, the total velocity field is approximated by

$$
\mathbf{V} \approx \overline{\mathbf{U}}+G(z) \mathbf{v}_{1}+G(2 z) \mathbf{v}_{2} ; \quad w \approx-\frac{H_{\mathrm{T}}}{\pi}\left[G^{\prime}(z) \operatorname{div} \mathbf{v}_{1}+\frac{1}{2} G^{\prime}(2 z) \operatorname{div} \mathbf{v}_{2}\right]
$$

where $\mathbf{V}$ is the horizontal velocity and $w$ is the vertical velocity. The total potential temperature is given approximately by

$$
\Theta \approx z+G^{\prime}(z) \theta_{1}+2 G^{\prime}(2 z) \theta_{2} .
$$

Here, $H_{\mathrm{T}} \approx 16 \mathrm{~km}$ is the height of the tropical troposphere with $0 \leq z \leq H_{\mathrm{T}}$ and $\mathbf{v}_{j}^{\perp}=\left(-v_{j}, u_{j}\right)$ while $\overline{\mathbf{U}}$ is the incompressible barotropic wind which is set to zero here for simplicity and is added to the equations above only for illustration and for the sake completeness and consistency with other crude vertical resolution models. This actually clarifies the fact that the background vertical mean flow is removed from the equations. In (2.1), $P \geq 0$ models the heating from deep convection while $H_{\mathrm{s}}, H_{\mathrm{c}}$ are the stratiform and congestus heating rates. Conceptually, the direct heating mode has a positive component and serves to heat the whole troposphere and is associated with a vertical shear flow on either side of the heating. The second baroclinic mode is heated by the congestus heating, $H_{\mathrm{c}}$, from below and by the stratiform heating, $H_{\mathrm{s}}$, from above and therefore cooled by $H_{\mathrm{c}}$ from above and by $H_{\mathrm{s}}$ from below. It is associated with a jet shear flow in the middle troposphere on either side of the heating. The terms $S_{1}$ and $S_{2}$ are the radiative cooling rates associated with the first and second baroclinic modes, respectively.

The system of equations in (2.1) is augmented by an equation for the boundary layer equivalent potential temperature, $\theta_{\mathrm{eb}}$, and another for the vertically integrated moisture content, $q$.

$$
\begin{aligned}
& \frac{\partial \theta_{\mathrm{eb}}}{\partial t}=\frac{1}{h_{\mathrm{b}}}(E-D) \\
& \frac{\partial q}{\partial t}+\overline{\mathbf{U}} \cdot \nabla q+\operatorname{div}\left(\left(\mathbf{v}_{1}+\tilde{\alpha} \mathbf{v}_{2}\right) q+\tilde{Q}\left(\mathbf{v}_{1}+\tilde{\lambda} \mathbf{v}_{2}\right)\right)=-P+\frac{1}{H_{\mathrm{T}}} D .
\end{aligned}
$$

In (2.2), $h_{\mathrm{b}} \approx 500 \mathrm{~m}$ is the height of the moist boundary layer while $\tilde{Q}, \tilde{\lambda}$, and $\tilde{\alpha}$ are parameters associated with a prescribed moisture background and perturbation vertical profiles. According to the first equation in (2.2), $\theta_{\mathrm{eb}}$ changes in response to the downdrafts, $D$, and the sea surface evaporation $E$. The troposphere moisture equation for $q$ is derived (see Khouider and Majda, 2006a) 
from the bulk water vapor budget equation by imposing a moisture stratification-like background vertical profile $q^{\text {tot }}=Q(z)+q$. The approximate numerical values of $\tilde{\lambda}=0.8$ and $\tilde{\alpha}=0.1$, follow directly from the derivation, while the coefficient $\tilde{Q}$ arises from the background moisture gradient. We use the standard value $\tilde{Q} \approx 0.9$ (Yano and Emanuel, 1991; Frierson et al., 2004). The parameterizations in (2.1) and (2.2) automatically have conservation of an approximation to vertically integrated moist static energy (Khouider and Majda, 2006a).

The equations in (2.1) and (2.2) for the prognostic variables $q, \theta_{\mathrm{eb}}, \theta_{j}, \mathbf{v}_{j}, j=1,2$, are written in nondimensional units where the equatorial Rossby deformation radius, $L_{\mathrm{e}} \approx 1500 \mathrm{~km}$ is the length scale, the first baroclinic dry gravity wave speed, $c \approx 50 \mathrm{~m} \mathrm{~s}^{-1}$, is the velocity scale, $T=L_{\mathrm{e}} / c \approx 8 \mathrm{~h}$ is the associated time scale, and the dry-static stratification $\bar{\alpha}=\frac{H_{\mathrm{T}} N^{2} \theta_{0}}{\pi g} \approx 15 \mathrm{~K}$ is the temperature unit scale. The basic bulk parameters of the model are listed in Table 1 for the reader's convenience.

\subsection{The convective parametrization}

The surface evaporative heating, $E$, in (2.2) obeys an adjustment equation toward the boundary layer saturation equivalent potential temperature, $\theta_{\mathrm{eb}}^{*}$,

$$
\frac{1}{h_{\mathrm{b}}} E=\frac{1}{\tau_{\mathrm{e}}}\left(\theta_{\mathrm{eb}}^{*}-\theta_{\mathrm{eb}}\right)
$$

with $\tau_{\mathrm{e}}$ is the evaporative time scale. The value of $\theta_{\mathrm{eb}}^{*}$ on a warm ocean surface is fixed such that at radiative convective equilibrium (RCE) we have $\theta_{\mathrm{eb}}^{*}-\bar{\theta}_{\mathrm{eb}}=10 \mathrm{~K}$, according to the Jordan sounding (Gill, 1982). Notice that the precise values of $\theta_{\mathrm{eb}}^{*}, \bar{\theta}_{\mathrm{eb}}, \bar{\theta}_{\mathrm{em}}$ are not used here. Only the discrepancies $\theta_{\mathrm{eb}}^{*}-\bar{\theta}_{\mathrm{eb}}$ and $\bar{\theta}_{\mathrm{eb}}-\bar{\theta}_{\mathrm{em}}$ are needed below to set a radiative convective equilibrium (RCE).

Besides the second baroclinic moisture advection in (2.2), the originality of the present model resides in a new treatment of the deep-convective heating/precipitation, $P$, and the downdrafts, $D$, as well as the introduction of the congestus heating, $H_{\mathrm{c}}$, into the $\theta_{2}$ equation. The middle

Table 1

Bulk constants in two layer mode model

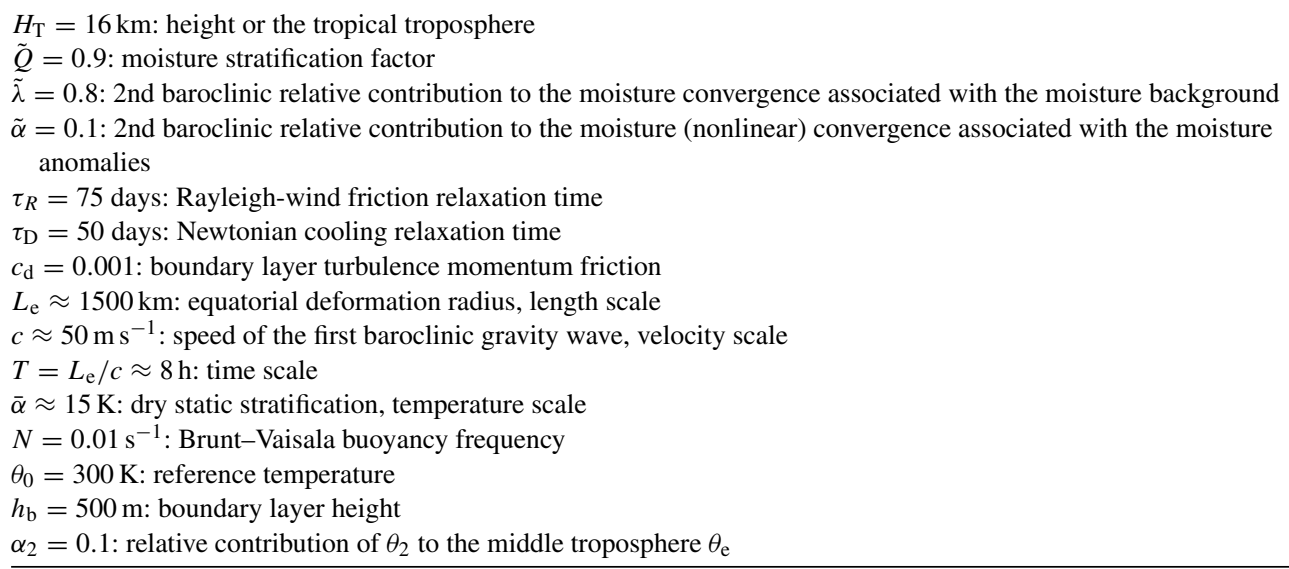


tropospheric equivalent potential temperature anomaly is defined approximately by

$$
\theta_{\mathrm{em}} \approx q+\frac{2 \sqrt{2}}{\pi}\left(\theta_{1}+\alpha_{2} \theta_{2}\right)
$$

where $\alpha_{2}=0.1$. Notice that the coefficient $2 \sqrt{2} / \pi$ in (2.4) results from the vertical average of the first baroclinic potential temperature, $\sqrt{2} \theta_{1} \sin \left(\pi z / H_{\mathrm{T}}\right)$, while the small value for $\alpha_{2}$ adds a nonzero contribution from $\theta_{2}$ to $\theta_{\mathrm{em}}$ to include a contribution from the lower middle troposphere although its vertical average is zero.

The model parametrization has a switch parameter $\Lambda$ which serves as a measure for the moistness and dryness of the middle troposphere (Zehnder, 2001; Khouider and Majda, 2006a). When the discrepancy between the boundary layer and the middle troposphere equivalent potential temperatures is above some fixed threshold, $\theta^{+}$, the atmosphere is defined as dry and we set $\Lambda=1$, leading to zero precipitation and a maximum fraction of downdrafts. When this discrepancy is below some lower value, $\theta^{-}$, we have a relatively moist atmosphere and we set $\Lambda=\Lambda^{*}<1$. The lower threshold $\Lambda^{*}$ can take any value between zero and one and here $\Lambda^{*}=0.2$ to ensure a minimal fraction of downdrafts when the atmosphere is moist and the precipitation is maximum. $\Lambda$ is then interpolated (linearly) between these two values. More precisely, we set

$$
\Lambda= \begin{cases}1 & \text { if } \theta_{\mathrm{eb}}-\theta_{\mathrm{em}}>\theta^{+} \\ A\left(\theta_{\mathrm{eb}}-\theta_{\mathrm{em}}\right)+B & \text { if } \theta^{-} \leq \theta_{\mathrm{eb}}-\theta_{\mathrm{em}} \leq \theta^{+} \\ \Lambda^{*} & \text { if } \theta_{\mathrm{eb}}-\theta_{\mathrm{em}}<\theta^{-} .\end{cases}
$$

Here, $\theta^{+}=20 \mathrm{~K}$ and $\theta^{-}=10 \mathrm{~K}$ while $A$ and $B$ are fitting constants guaranteeing continuity of $\Lambda$. The value of $\theta^{-}$is chosen according to the Jordan sounding (Fig. 3.5 from Gill, 1982). It represents a threshold below which the free troposphere is locally moist and "accepts" only deep convection.

Therefore, the precipitation, $P$, and the downdrafts, $D$, obey

$$
P=\frac{1-\Lambda}{1-\Lambda^{*}} P_{0} \quad \text { and } \quad D=\Lambda D_{0}
$$

while the stratiform and congestus heating rates, $H_{\mathrm{s}}$ and $H_{\mathrm{c}}$, solve the relaxation-type equations

$$
\frac{\partial H_{\mathrm{s}}}{\partial t}=\frac{1}{\tau_{\mathrm{s}}}\left(\alpha_{\mathrm{s}} P-H_{\mathrm{s}}\right)
$$

and

$$
\frac{\partial H_{\mathrm{c}}}{\partial t}=\frac{1}{\tau_{\mathrm{c}}}\left(\alpha_{\mathrm{c}} \frac{\Lambda-\Lambda^{*}}{1-\Lambda^{*}} \frac{D}{H_{\mathrm{T}}}-H_{\mathrm{c}}\right),
$$

respectively. Notice that, as anticipated above, when the middle troposphere is dry, $\Lambda=1$, deep convection is completely inhibited, even if $P_{0}$ (which plays the role of CAPE here) is positive, whereas congestus heating is favored. In the absence of deep convection, the downdrafts are interpreted as the subsidence associated with the detrainment of shallow clouds. In this sense, the congestus clouds serve to moisten and precondition the middle troposphere to sustain deep convection by lowering $\Lambda$ in the model via both the increase of $q$ and the decrease of $\theta_{\mathrm{eb}}$. The situation is inverted during the deep-convective episodes when $\Lambda=\Lambda^{*}$. Moreover, the dry atmosphere increases the downdrafts, $D$, and promotes boundary layer clouds.

The quantities $P_{0}$ and $D_{0}$ represent, respectively, the maximum allowable deep-convective heating/precipitation and downdrafts, independent of the value of the switch function $\Lambda$. Notice 
that conceptually the model is not bound to any type of convective parametrization. A Betts-Miller relaxation type parametrization as well as a CAPE parametrization can be used to setup a closure for $P_{0}$. Indeed, here we use a combination of the two concepts by letting

$$
P_{0}=\frac{1}{\tau_{\mathrm{conv}}}\left(a_{1} \theta_{\mathrm{eb}}+a_{2}(q-\hat{q})-a_{0}\left(\theta_{1}+\gamma_{2} \theta_{2}\right)\right)^{+}
$$

where $\hat{q}$ is a threshold constant value measuring a significant fraction of the tropospheric saturation and $\tau_{\text {conv }}, a_{1}, a_{2}, a_{0}$ are parameters specified below and in Table 2 (Fuchs and Raymond, 2002; Frierson et al., 2004). In particular, the coefficient $a_{0}$, which is the inverse buoyancy relaxation time of Fuchs and Raymond (2002), is an important parameter to vary and its role is studied in the next section. Notice that the precise value of $\hat{q}$ is not important here. With $\bar{\theta}_{1}=\bar{\theta}_{2}=0$ at RCE, we define and use the bulk quantity $\bar{P}_{0}=\frac{1}{\tau_{\text {conv }}}\left(a_{1} \bar{\theta}_{\mathrm{eb}}+a_{2}(\bar{q}-\hat{q})\right)=\left(1-\Lambda^{*}\right) /(1-\bar{\Lambda}) \bar{P}$ which is the maximum allowable precipitation at RCE and its value is given in Table 2. $P$ is hence rewritten as

$$
P=\frac{1-\Lambda}{1-\Lambda^{*}}\left(\bar{P}_{0}+\frac{1}{\tau_{\mathrm{conv}}}\left(a_{1} \theta_{\mathrm{eb}}^{\prime}+a_{2} q^{\prime}-a_{0}\left(\theta_{1}^{\prime}+\gamma_{2} \theta_{2}^{\prime}\right)\right)\right)
$$

where the primed variables represent perturbations from RCE. The parameter $\gamma_{2}$, which couples $\theta_{2}$ to $P_{0}$ is also varied to assess the effect of the lower troposphere temperature variation on the parameterizations; a relatively warm lower troposphere will promote evaporation and detrainment of cumulus clouds. Thus, it should result in a weakening of the deep convection.

The downdrafts are closed by

$$
D_{0}=\frac{m_{0}}{\bar{P}}\left(\bar{P}+\mu_{2}\left(H_{\mathrm{s}}-H_{\mathrm{c}}\right)\right)^{+}\left(\theta_{\mathrm{eb}}-\theta_{\mathrm{em}}\right)
$$

where $m_{0}$ is a scaling of the downdraft mass flux and $\bar{P}$ is a prescribed precipitation/deepconvective heating rate at radiative convective equilibrium and appears here as a scaling factor.

Table 2

Parameters in the convective parameterization

$\theta_{\mathrm{eb}}^{*}-\bar{\theta}_{\mathrm{eb}}=10 \mathrm{~K}$ : discrepancy between the boundary layer saturation and the equivalent potential temperatures

$\bar{\theta}_{\mathrm{eb}}-\bar{\theta}_{\mathrm{em}}=14 \mathrm{~K}$ : discrepancy between boundary and middle tropospheric equivalent potential temperature at RCE

$m_{0}=1.9491 \times 10^{-5} \mathrm{~m} \mathrm{~s}^{-1}$ (depends on $Q_{R, 1}^{0}$ and $\bar{\theta}_{\mathrm{eb}}-\bar{\theta}_{\mathrm{em}}$ ): scaling of downdraft mass flux

$\bar{P}_{0}=\frac{1-\Lambda^{*}}{1-\bar{\Lambda}} \bar{P}=0.0667 \mathrm{~K} \mathrm{day}^{-1}$ : maximum allowable precipitation at RCE

$\tau_{\mathrm{e}} \approx 9$ days: evaporative time scale in the boundary layer

$\theta^{ \pm}=10,20 \mathrm{~K}$ : temperature thresholds used to define the switch function $\Lambda$

$\Lambda^{*}=0.2$ : lower threshold of the switch function $\Lambda$

$A, B$ : linear fitting constants interpolating the switch function $\Lambda$

$\tau_{\mathrm{s}}=3 \mathrm{~h}$ : stratiform heating adjustment time

$\alpha_{\mathrm{s}}=0.25$ : stratiform heating adjustment coefficient

$\tau_{\mathrm{c}}=1 \mathrm{~h}$ : congestus heating adjustment time

$\alpha_{\mathrm{c}}=0.5$ : congestus heating adjustment coefficient

$a_{0}=7.5$ (varies): inverse buoyancy time scale of convective parametrization

$a_{1}=0.1$ : relative contribution of $\theta_{\mathrm{eb}}$ to the convective parametrization

$a_{2}=0.9$ : relative contribution of $q$ to the convective parametrization

$\tau_{\text {conv }}=2 \mathrm{~h}$ : deep-convective reference time scale

$\gamma_{2}=1$ : relative contribution of $\theta_{2}$ to the convective parametrization (strength of lower troposphere coupling)

$\mu_{2}=0.5$ : relative contribution of stratiform and congestus mass flux anomalies

Values are consistent with both the Jordan sounding and the model's RCE. 
Here, $\mu_{2}$ is a parameter allowing for stratiform and congestus downward mass flux anomalies (MS; MKKSS) through cooling from evaporation of stratiform rain in the lower troposphere. This flux increases with increasing stratiform heating and decreases with increasing congestus heating which warms the lower troposphere, partly compensating the evaporative cooling. The plus sign in the upper script ensures that $D_{0}$ is positive whenever $\theta_{\mathrm{eb}}>\theta_{\mathrm{em}}$ thus the downdrafts are bringing lower $\theta_{\mathrm{e}}$ air into the boundary layer. Although $\mu_{2}$ contributes only by a small amount to the convective instability from linear theory, in the present model (results not shown), we expect this contribution to play an important role in nonlinear simulations since the stratiform downward mass flux is essential for the stratiform instability (MS, MKKSS) in triggering the regeneration of $\theta_{\mathrm{eb}}$ after the passage of deep convection.

Finally, the radiative cooling rates, $S_{1}, S_{2}$ in (2.1) are given by a simple Newtonian cooling model

$$
S_{j}=-\frac{\pi}{2 \sqrt{2}} Q_{R, j}^{0}-\frac{1}{\tau_{\mathrm{D}}} \theta_{j}, \quad j=1,2
$$

where $Q_{R, j}^{0}, j=1,2$ are the radiative cooling rates at RCE. The basic constants in the model convective parametrization and the typical values utilized here are given in Table 2 . The physical features of the present multi-cloud model parametrization are discussed extensively in Khouider and Majda (2006a).

\subsection{Linear stability analysis at RCE}

A standard tool in understanding the basic properties of a convective parametrization is the linearized stability analysis at radiative convective equilibrium (Emanuel, 1987; Yano et al., 1998; Majda and Shefter, 2001a; MS; Fuchs and Raymond, 2002). A radiative convective equilibrium (RCE), which is a state where the convective heating is balanced by the radiative cooling, is a time independent, static, and spatially homogeneous solution to the set of Eqs. (2.1)-(2.11) described above. It sets up a steady state solution around which convective waves can oscillate and grow. Here, we setup an RCE with all parameter values as in Table 2 for the radiative cooling $Q_{R, 1}^{0}=0.04 \mathrm{~K} \mathrm{day}^{-1}$ and report the results of linearized stability analysis as the inverse buoyancy relaxation time $a_{0}$ in (2.9) varies. All the details of the procedure are omitted and the interested reader can consult Khouider and Majda (2006a) for further details. Note that $\bar{\theta}_{1}, \bar{\theta}_{2}$ are set to zero at RCE so that $\bar{P}=Q_{R, 1}^{0}$.

In Fig. 1, we show the phase speed on the left and the growth rate on the right as a function of the zonal wavenumber for the linear waves in the multi-cloud parametrization about the RCE for the varying values, $a_{0}=1,5,7.5$, and 10 . As the reader can see, the unstable wavenumbers typically are associated with waves with phase velocities between $15-20 \mathrm{~m} \mathrm{~s}^{-1}$ and have instabilities confined to large scale of order $1000 \mathrm{~km}$ and larger although the exceptional case with $a_{0}=1$ has instability down to $500 \mathrm{~km}$. As shown in Khouider and Majda (2006a), these instabilities have the physical structure of convectively coupled waves.

Except for $\gamma_{2}$ and $a_{0}$, the other convective parameters, namely as $\alpha_{2}, \mu_{2}$ and $a_{1}, a_{2}$ under the constraint $a_{1}+a_{2}=1$, have a secondary effect on linear stability results (Khouider and Majda, 2006a). Nevertheless, we keep them nonzero here for the sake of completeness because they represent natural physical effects which we believe are important during the different stages of the large scale convective life cycle as described below. 

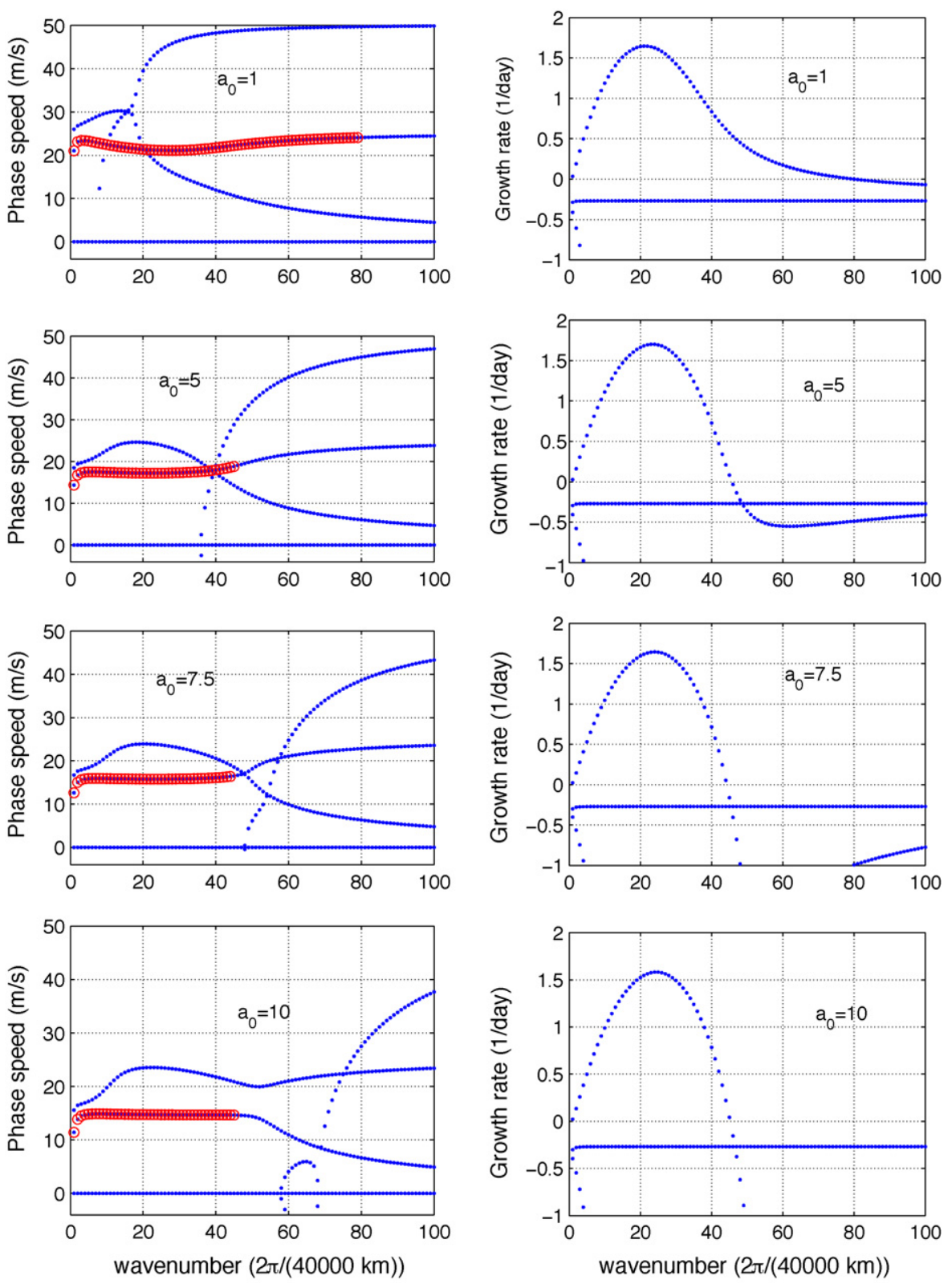

Fig. 1. Phase speed (left) and growth rate (right) as a function of the wavenumber for increasing values of the inverse buoyancy relaxation time parameter $a_{0} . \alpha_{2}=0.1, \gamma_{2}=1, \mu_{2}=0.5, \tilde{\lambda}=0.8, a_{1}=0.1, a_{2}=0.9$, and $\theta_{\mathrm{eb}}-\theta_{\mathrm{em}}=14 \mathrm{~K}$. Because of symmetry, only the first quadrant is shown for the dispersion relation diagram and the branches with positive growths are highlighted with small circles. 


\section{Nonlinear convectively coupled gravity waves}

\subsection{Discretization and setup for nonlinear simulations}

The system of equations described by (2.1)-(2.11) has the form

$$
\frac{\partial U}{\partial t}+\operatorname{div}(F(U))=B(U)
$$

where $U$ is a vector valued function of $(x, t)$ representing the diagnostic variables $\mathbf{v}_{1}, \mathbf{v}_{2}, \theta_{1}, \theta_{2}, \theta_{\mathrm{eb}}, q, H_{\mathrm{s}}$, and $H_{\mathrm{c}}$, while $F(U)$ represents the conservative terms in (2.1) and (2.2), and $B(U)$ stands for the linear and nonlinear terms on the right hand sides obtained through the various diagnostic and prognostic equations in (2.3)-(2.11). Our basic discretization strategy consists of a fractional time splitting procedure where the conservative part and the forcing part, $\frac{\partial U}{\partial t}+\operatorname{div}(F(U))=0$ and $\frac{\partial U}{\partial t}=B(U)$, respectively, are solved independently during one time step. In order to preserve the second order accuracy in time during the splitting, we adopt Strang's well known strategy (Khouider and Majda, 2005a,b). The conservative part is solved by a second order (conservative) finite volume (central) scheme while the forcing part, which reduces to a simple ODE system, is handled by a second order Runge-Kutta method (Khouider and Majda, 2005a,b).

The numerical simulations presented below are initialized using the mixed deep-convective congestus RCE, discussed earlier in Section 2.3 and Table 2 and involve the homogeneous solution plus a small inhomogeneous perturbation. Rotation is ignored for simplicity, so that many of the complex equatorial wave dynamics are filtered out leaving only the gravity waves with the possibility of traveling in the both east-west directions with complete symmetry. The resulting system of equations in $(x, t)$ space is then solved on a periodic domain whose length $X=40,000 \mathrm{~km}$, mimicking the equatorial circumference of the earth, and run for several hundred days.

\subsection{Effect of the inverse buoyancy relaxation time $a_{0}$ on convective activity}

To investigate the effect of the inverse buoyancy relaxation time parameter, $a_{0}$, we plot in Fig. 2 the histories of root mean square (rms) of the first baroclinic velocity component, $\left[\frac{1}{X} \int_{0}^{X}\left(u_{1}(x, t)\right)^{2} \mathrm{~d} x\right]^{1 / 2}$ for increasing values of $a_{0}: a_{0}=1,5,10$, and 20. Clearly, the parameter $a_{0}$ plays a central role in setting both the strength and the frequency of the large scale deepconvective events, which, as we will see below, are associated with the bursts in the $u_{1} \mathrm{rms}$. At $a_{0}=1$, we see only two giant $u_{1}$ rms peaks reaching nonphysically large values exceeding $20 \mathrm{~m} \mathrm{~s}^{-1}$ over the integration period of 1200 days. The two peaks are separated by a quiet period of more than 800 days. The convective event itself does not last more than 100 days. As $a_{0}$ increases the strength of the peaks decreases drastically while the frequency of the convective events increases. Especially, when $a_{0}=5$, the rms peaks do not exceed the reasonable value of $4 \mathrm{~m} \mathrm{~s}^{-1}$ and they roughly occur at a 100-day period with the quiet-motionless episodes lasting roughly as much as the convective events. Although, there is apparently a well determined time scale on which the convective episodes occur, the strength and shape of the bumps seem to be chaotic with some peaks being much weaker than others. When $a_{0}=10$, the plot becomes very noisy and the overall amplitude is much weaker. We can hardly see motionless periods as sometimes, if not most of the times, the next convective event seems to go up before the motion from the previous one stops completely. At $a_{0}=20$, the convective motion does not shut off completely 
at any time and the overall amplitude of the $u_{1}$ rms becomes very weak. We notice also that, with $a_{0}=20$, the simulation approaches a quasi-steady state where the oscillations become smooth and periodic.

\subsection{Convective organization: intermittent deep-convective events and nonlinear convectively coupled waves}

Now we focus on the case $a_{0}=7.5$ to explore and analyze in full detail the structure of the resulting nonlinear convectively coupled waves in the multi-cloud convective parametrization. Notice that $a_{0}=7.5$ is intermediate between the moderate regime of $a_{0}=5$ and the more chaotic and slightly weak amplitude regime at $a_{0}=10$, in Fig. 2. First, we present in Fig. 3 the rms histories of all the prognostic variables, $u_{1}, u_{2}, \theta_{1}, \theta_{2}, \theta_{\mathrm{eb}}, q, H_{\mathrm{s}}$, and $H_{\mathrm{c}}$. The congestus heating, $H_{\mathrm{c}}$, is rescaled by a factor 20 for clarity. Since $H_{\mathrm{s}}$ obeys a $3 \mathrm{~h}$ (only) adjustment scheme toward the deep-convective heating, $P$, it can be used as a surrogate for deep-convective events. Notice that all the eight variables are more or less correlated except for the congestus heating. Also while $u_{1}, u_{2}, \theta_{1}$, and $\theta_{2}$ seem to be in phase, the peaks of $\theta_{\mathrm{eb}}$ are always leading the convective events and followed by those of $q$.

Now we concentrate on the time period 1050-1160 days highlighted on all the panels in Fig. 3. Notice that two consecutive peaks of different shapes are included. In Fig. 4, we display the space-time contours of $u_{1}, u_{2}, \theta_{\mathrm{eb}}, q, H_{\mathrm{s}}$, and $H_{\mathrm{c}}$ over the period highlighted in Fig. 3 on one full period of the spatial domain. This demonstrates that indeed the chaotic behavior seen in the time series is associated with a highly intermittent nonlinear convectively coupled gravity wave activity. Apparent from all the six panels are two different large scale events of large variance separated by a calm period of roughly 20 days. The contours of $H_{\mathrm{s}}$ indicate implicitly the regions in the space-time plane of deep-convective activity associated with each event. The first convective event starts at around day 1070 and ends at around day 1100 while the second convective event starts approximatively at day 1120 and ends at around day 1150 . The two events seem to be disjoint in all the presented variables except for the contours of $u_{2}$ and somewhat $q$ which seem to connect the organized large scale convective episodes. Indeed, after a short turbulent period starting a few days before day 1070 , as seen particularly in the $H_{\mathrm{s}}$ contours, two gravity waves are ejected in the opposite directions. The two gravity waves are visible on all six panels. However, only the westward propagating wave amplifies and survives from what seems to otherwise result
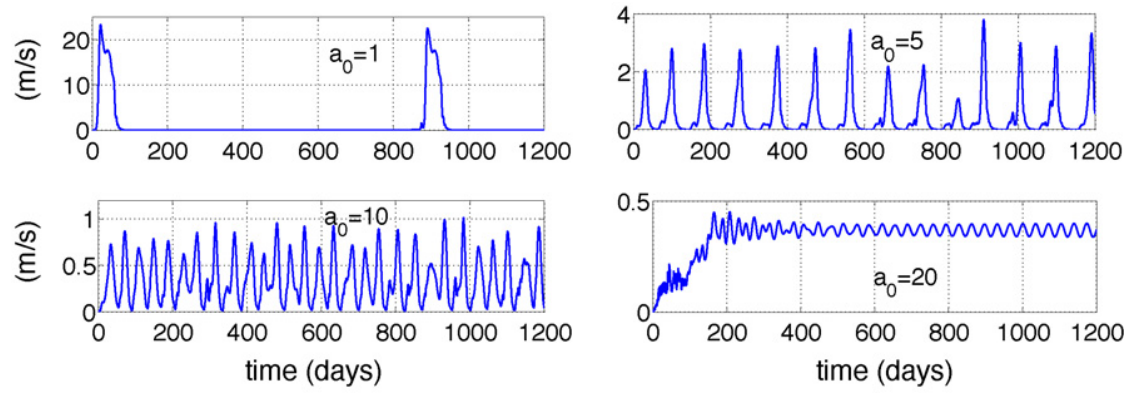

Fig. 2. History of the root mean square of the first baroclinic velocity component, $u_{1}$, for different values of $a_{0}$ demonstrating the nontrivial effect on the strength and intermittency of convective activity. The values of $a_{0}$ are indicated and the other parameters are as in Fig. 1. 

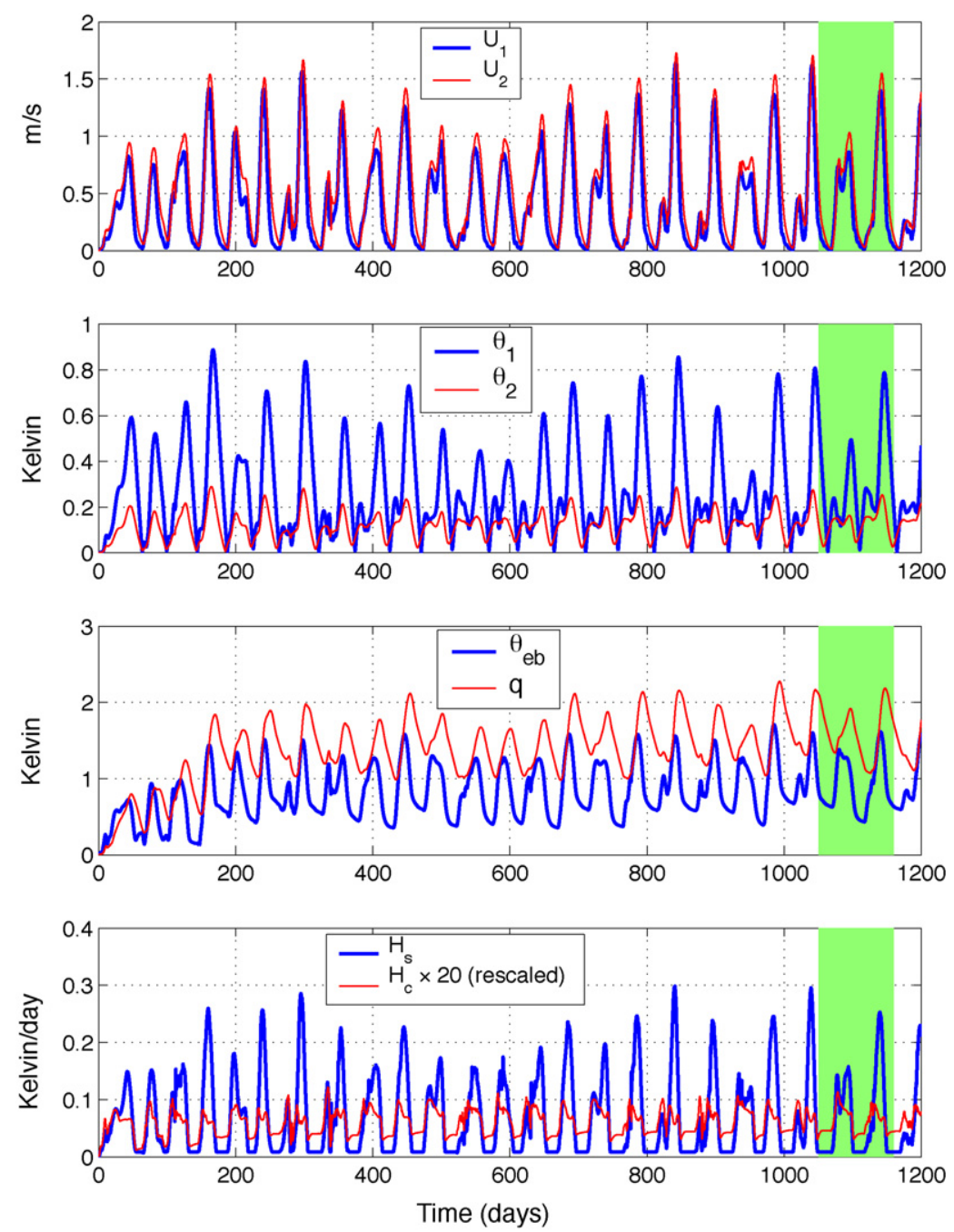

Fig. 3. History of the root mean square of all the prognostic variables, $u_{1}, u_{2}, \theta_{1}, \theta_{2}, \theta_{\mathrm{eb}}, q, H_{\mathrm{s}}$, and $H_{\mathrm{c}}$, for the case $a_{0}=7.5$. The other parameters are as in Fig. 1. Notice the highlighted time window from 1050 to 1160 days.

in a collision between the two waves near the edge of the periodic domain. The reasons for the extinction of the weaker wave are not very clear at this stage but it is plausibly due to the fact that the atmosphere is relatively dry away from the deep-convective region associated with the strong wave, thanks to the first and second baroclinic moisture convergence, thus forcing convective organization toward the strongest deep-convective pockets. The stronger wave continues to amplify and then decays. Finally, the deep-convective activity ceases at around time 1100 days. The gravity wave then rides on the second baroclinic mode increasing its propagation speed, as it remains visible (only) on the $U_{2}$ panel in Fig. 4. After time 1100 days, the wave does not carry heating, $u_{1}$, or $\theta_{\mathrm{eb}}$ components anymore and is essentially a dry second baroclinic gravity wave except for the weak congestus anomalies it carries. However, the moisture convergence/divergence in this wave generates moisture anomalies moving with the wave. The two convective events, 

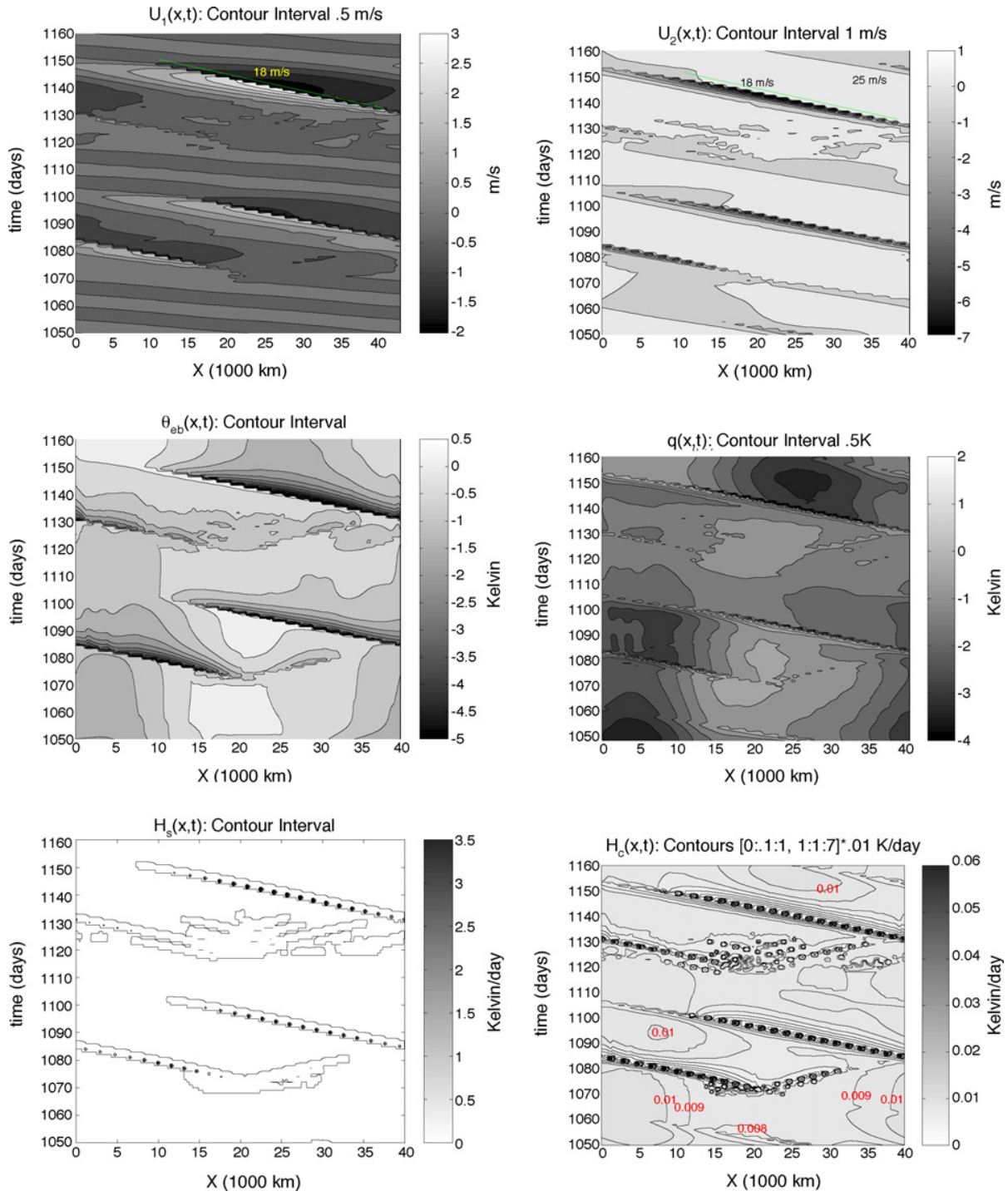

Fig. 4. $(x, t)$ contours of $u_{1}, u_{2}, \theta_{\mathrm{eb}}, q, H_{\mathrm{s}}$, and $H_{\mathrm{c}}$ showing the signature of the wave tracks over the time window highlighted in Fig. 3.

depicted in Fig. 4, follow more or less the same pattern. This pattern begins by: (i) a convective turbulent stage where moist gravity waves are ejected in both directions; (ii) after a while, all the convection 'converges' toward the strongest wave which persists and amplifies; (iii) the resulting convectively coupled wave propagates and gets engaged in a decaying phase leading to the demise of the deep-convective activity; (iv) then, the wave rides on a faster track as a second baroclinic dry/congestus gravity wave.

Indeed, as pointed out on the top of the $U_{2}$ panel and also the $U_{1}$ panel in Fig. 4, the (deep) convectively coupled wave move at a speed of roughly $18 \mathrm{~m} \mathrm{~s}^{-1}$ while its congestus counterpart (continuation) moves at roughly $25 \mathrm{~m} \mathrm{~s}^{-1}$; the speed of the dry second baroclinic gravity wave. 
Notice that, although weak, the second baroclinic continuation of the wave carries a significant trace of moisture, $q$, perturbation, due to the second baroclinic convergence term, $\tilde{\lambda} \operatorname{div} \mathbf{v}_{2}$, present in the $q$ equation. Moreover, the $U_{1}$ contours seem to follow a much faster track, a $50 \mathrm{~m} \mathrm{~s}^{-1}$ dry gravity wave, right after or even before the extinction of the deep-convection which very quickly decays and becomes nearly insignificant (see Fig. 6(D) below).

Interestingly, the intermittent convection and wave propagation activity of Fig. 4 resemble the observed wave tracks of convectively coupled Kelvin waves reported in the literature such as the filtered OLR contours in Fig. 3 of Straub and Kiladis (2002) and Fig. 9(b) of Wheeler and Kiladis (1999), including the propagation speed of $18 \mathrm{~m} \mathrm{~s}^{-1}$ and the length scale of the convective region of about 1000-2000 km. They are identified as eastward propagating envelopes/convective super-clusters (Nakazawa, 1988; Wheeler and Kiladis, 1999; Wheeler et al., 2000; Straub and Kiladis, 2002; MKKSS).

In fact, in Fig. 5, we plot the local two-dimensional $(x-z)$ structure of the total convective heating field, $P(x, t) \sin \left(\frac{\pi z}{H_{\mathrm{T}}}\right)+2\left(H_{\mathrm{c}}(x, t)-H_{\mathrm{s}}(x, t)\right) \sin \left(2 \frac{\pi z}{H_{\mathrm{T}}}\right)$ (top panel) and the potential temperature, $\Theta(x, z, t)$ (middle) contours with the velocity field, $(U(x, z, t), w(x, z, t))$, profile overlaid on top of them (and on the bottom panel for clarity), at time $t=1095$ days during the mature stage of the first convective event of Fig. 4. Notice that the $x$-axis is reversed so that the convective wave can be easily compared to an observed eastward propagating Kelvin wave. Similarly to the results in MKKSS, the structure of convectively coupled wave in Fig. 5 has many features qualitatively resembling those of the observed convectively coupled Kelvin wave, in the lower atmosphere (below the tropopause), as depicted, for example, in Figs. 1-3 of MKKSS, including the strong upward flow within the deep-convective region, a similar vertical tilt in the zonal velocity and temperature contours, with cold anomalies in the lower troposphere and warm temperature aloft within and slightly leading the deep-convective region, exhibiting more resemblance with the observations than its linear counterpart shown in Fig. 6 of Khouider and Majda (2006a), and the stratiform heating trailing the wave. Also the magnitude of temperature perturbations of about 1-2 degrees, the maximum zonal velocity of $7 \mathrm{~m} \mathrm{~s}^{-1}$, and the maximum vertical velocity of $11 \mathrm{~cm} \mathrm{~s}^{-1}$, are all in a reasonable range of values as seen in the observations (MKKSS; Straub and Kiladis, 2002).

\subsection{The large scale convective cycle}

In an attempt to understand the physical mechanisms underlying the convective activity of the present multi-cloud convective parametrization, we plot in Fig. 6 the zonal structure of the zonal velocity components, $u_{1}, u_{2}$, the heating components, $P, P_{0}, H_{\mathrm{s}}, H_{\mathrm{c}}$, the switch parameter $\Lambda$, and the thermodynamic quantities involved in the expression of $P_{0}$ in (2.9), $\theta_{\mathrm{eb}}, q$ and $\theta_{1}+\gamma_{2} \theta_{2}$, at four different times associated with the first convective event of Fig. 3. During the birth stage of deep convection at time $t=1070$ days, during the mature stage at time $t=1094$ days, during the decaying stage at time $t=1100$ days, and a few days after the complete extinction of the deep-convective wave at time $t=1110$ days.

The deep-convective activity starts during the birth stage as an aggregate or a cluster of random and somewhat isolated deep-convective sites as seen in the heating plot of Fig. 6(A) at time $t=$ 1070 days. A somewhat turbulent flow is associated with this scattered heating spots. Moreover, in agreement with physical intuition, the convective aggregate is localized in the region of relatively weak $\Lambda$, high $\theta_{\mathrm{eb}}$, and high $q$. Notice also the slightly anomalously negative $\theta_{1}+\gamma_{2} \theta_{2}$ over the entire domain at time $t=1070$ days. Then, the convection organizes itself into two moist gravity waves moving in opposite directions. As it is pointed above, ultimately only the strongest wave 

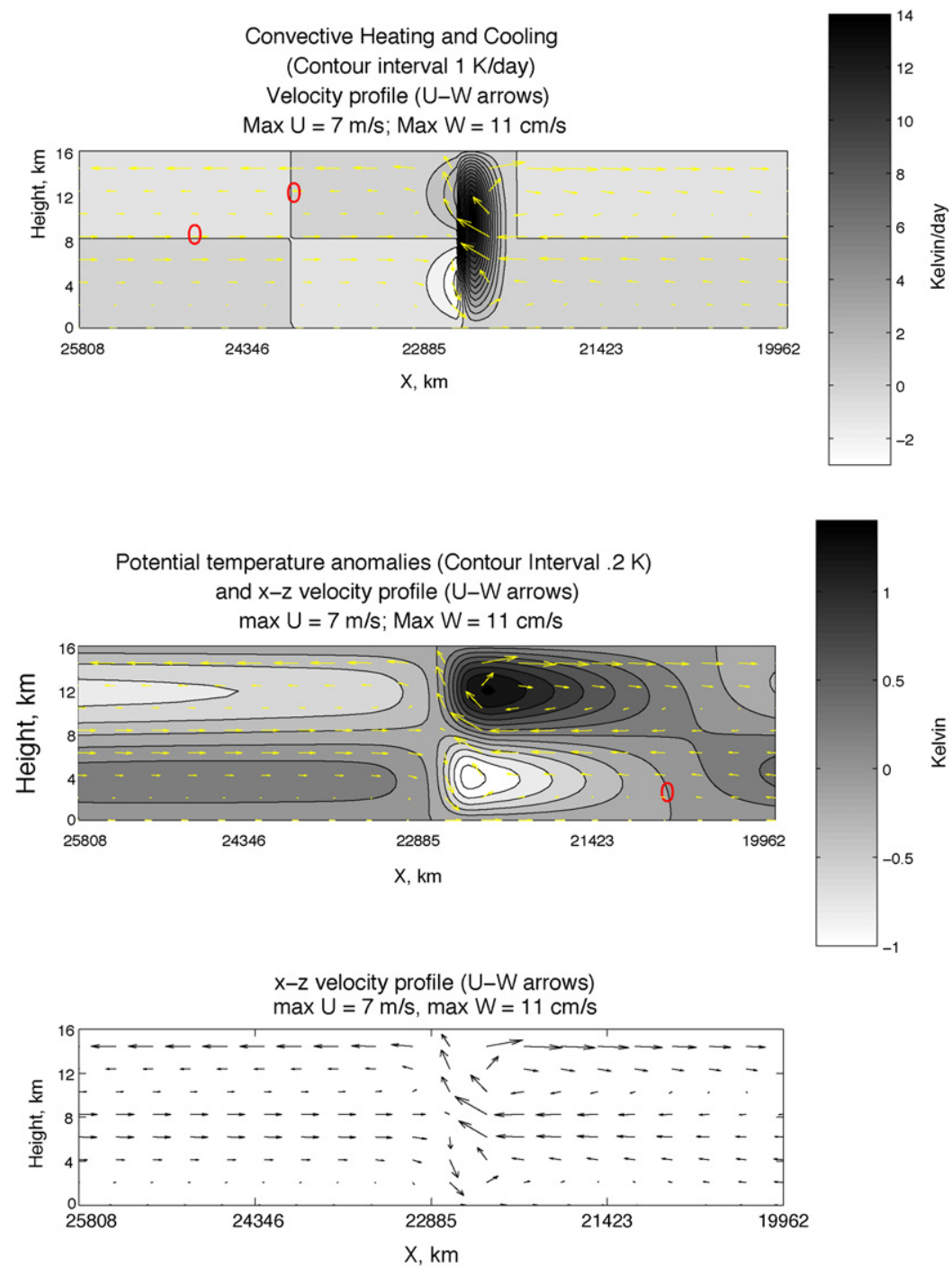

Fig. 5. Local structure of the convectively coupled wave during the mature stage at time $t=1095$ days moving at roughly $18 \mathrm{~m} \mathrm{~s}^{-1}$ starting around day 1070 and extinguishing approximatively at day 1100 from Fig. 4 . Notice that the $x$-axis is reversed so that the wave structure can be directly compared to an observed (eastward moving) tropical Kelvin wave.

amplifies further and persists for a longer time period. The plots presented in Fig. 6(B) at time $t=1095$ days reveal the structure of this strongest wave at its mature stage, before it starts to decay. The heating rates and the velocity magnitudes are stronger by a factor of 50 or so (especially $u_{2}$ ) when compared to the birth stage and the quantity $\theta_{1}+\gamma_{2} \theta_{2}$ is now positive all over the domain thanks to the deep-convective heating dominating the strong first baroclinic convergence at the heating peak. Furthermore, a wave pattern is clearly identified by following the steep/shock-like profile seen in all the variables. For the example in Fig. 6(B), this is characterized by the positive heating peak centered roughly around $x=22,500 \mathrm{~km}$. The shocks in $u_{1}$ and $u_{2}$ which are more or 
less in phase with the heating peak ( $u_{2}$ is slightly lagged) result from the strong upward motion associated with the convective activity and help create the shock in $\theta_{1}+\gamma_{2} \theta_{2}$. The abrupt decrease in moisture $q$ and $\theta_{\mathrm{eb}}$ result, respectively, from the drying of the troposphere through precipitation and the strong convective downdrafts resulting from the evaporation of stratiform rain.

Notice that an increase in $\theta_{\mathrm{eb}}$, on the left of the shock, leads the wave and is followed by a sudden increase in the moisture $q$ which yields a significant decrease in the switch parameter $\Lambda$ allowing a substantial amplification of deep-convection. The dying stage is presented in Fig. $6(\mathrm{C})$ at time $t=1100$ days. During this decaying stage, the deep-convective heating, and the anomalous $u_{1}, u_{2}, \theta_{\mathrm{eb}}$, and to some extent $q$ become abruptly weak due to CAPE consumption and drying, except for the second baroclinic disturbance, $u_{2}$, which also decays significantly but remains relatively high and persists beyond the demise of the deep-convective activity. The second baroclinic anomalies are carried by the second baroclinic (dry) gravity wave as shown in Fig. 4 . Notice from Fig. 6(C), the moisture trough in $q$ is plunging down to $-2 \mathrm{~K}$ at time $t=1100$ days around $x=5 \mathrm{~km}$ while $\theta_{\mathrm{eb}}$ is below $-1 \mathrm{~K}$ at some locations. Fig. 6(D) presents the same quantities
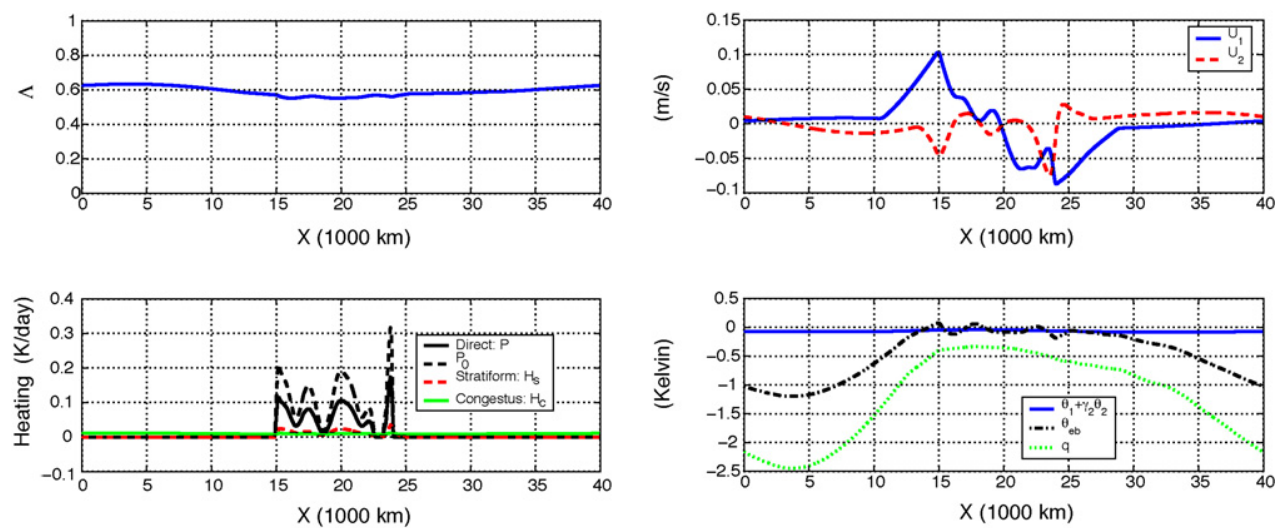

(A)

Birth stage ; time 1070 days
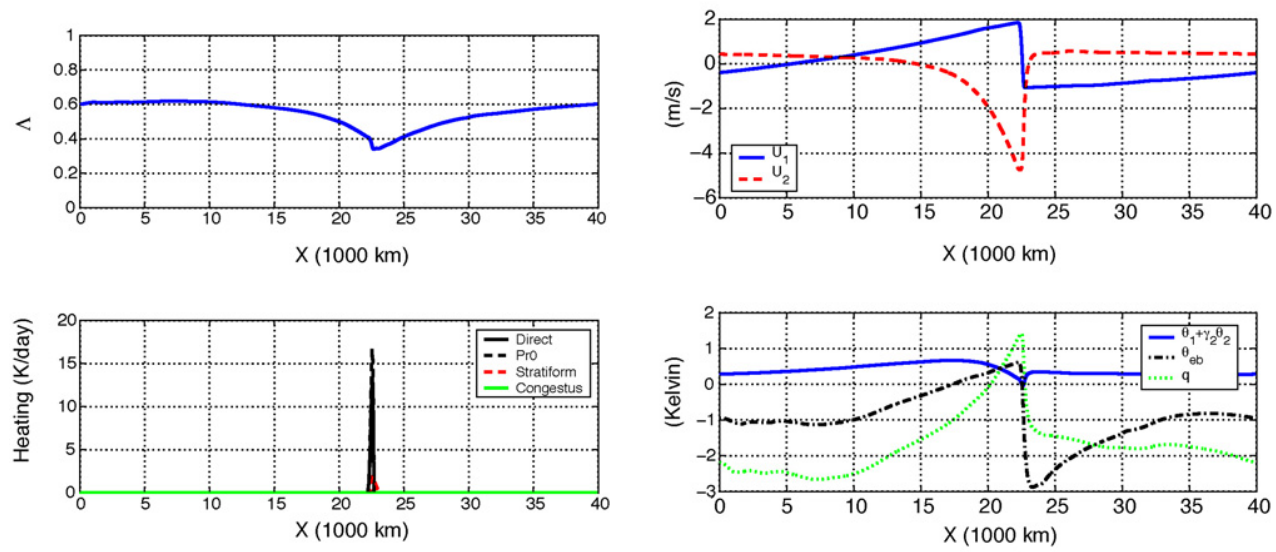

(B)

Mature stage; time 1095 days

Fig. 6. (A-D) Four time snapshots of the zonal structure of the prognostic and heating anomalies at four different episodes representative of the large scale convective cycle. 

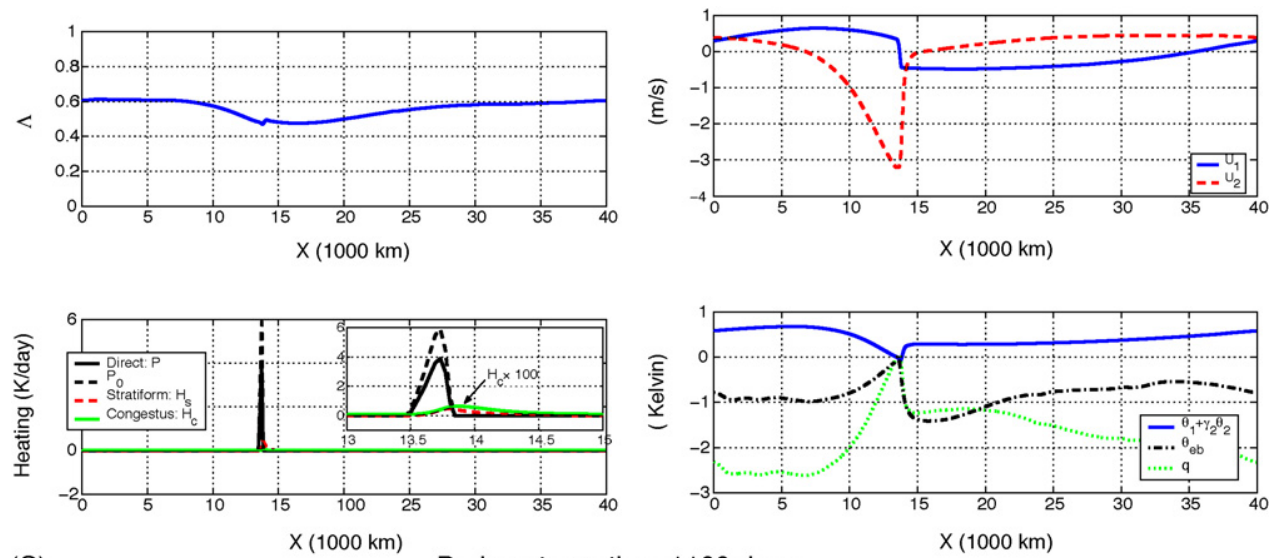

(C)

Drying stage; time 1100 days
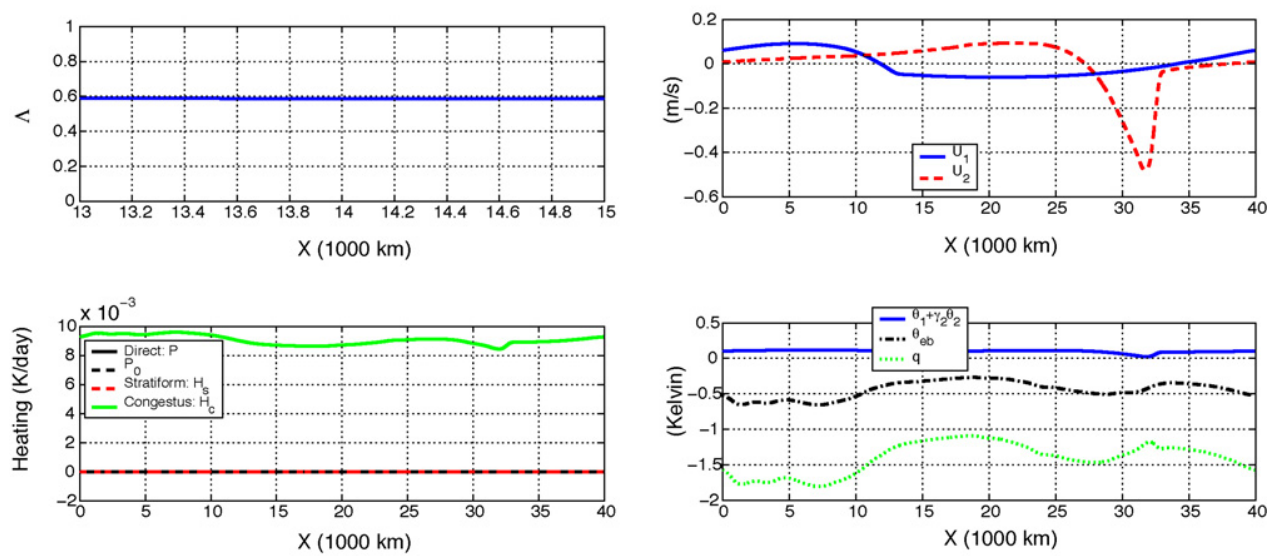

(D)

Congestus-preconditioning stage; time 1110 days

Fig. 6. (Continued).

10 days after, i.e, at time $t=1110$ days. As one would expect the deep convection is completely shut off but the congestus heating although weak remains active everywhere in the domain with an apparent kink associated with the strong $u_{2}$ gradient. Recall from (2.8) that the congestus heating adjusts to a significant fraction of the downdrafts $D$ in regions where the atmosphere is dry $(\Lambda \approx 1)$. In the absence of deep convection, the downdrafts $D$ can be thought of as the effect of evaporative cooling from the detrainment of congestus and nonprecipitating shallow clouds. Therefore, it has the double effect of moistening the upper atmosphere and cooling the boundary layer. It is this moistening effect which helps restore the moisture level as seen at time $t=1110$ days, when $D$ acts alone while $P=0$; thus, this period of time between the total demise of deep convection and the generation of the next convective episode is called the preconditioning stage. The mean value of $\theta_{\mathrm{eb}}$ is also increased a great deal over that period, especially right after the deep-convective episode, thanks to the action of the surface evaporative rate $E$ (relaxing $\theta_{\mathrm{eb}}$ toward a uniform sea surface saturation $\theta_{\mathrm{e}}$ ) when the downdrafts are weak, as a result of both the weak $\theta_{\mathrm{eb}}$ and the relatively high $\theta_{\mathrm{em}}$ (from the contribution of restored $\theta_{1}+\alpha_{2} \theta_{2}$ anomalies, not shown). 
From Fig. 4, the propagation of the 2 nd baroclinic gravity wave is evident during the preconditioning period. In the absence of a positive heating, the combination $\theta_{1}+\gamma_{2} \theta_{2}$ is subjected to a homogeneous radiative cooling and decreases uniformly. For the weaker radiative cooling, $Q_{R, 1}^{0}=0.04 \mathrm{~K} \mathrm{day}^{-1}$ in the present study, this process takes several days. Notice also the small bump in the moisture plot which seems to be carried by the second baroclinic convergence. These are the main mechanisms which precondition the atmosphere and prepare it for the next deep-convective event. If we now go back to the birth stage in Fig. 6(A), we see clearly that the convective activity starts at the moment when and location where the 2 nd baroclinic congestus gravity wave hits the region of high $\theta_{\mathrm{eb}}$ and high $q$. Therefore, we conclude that the small amount of moisture carried by the second baroclinic convergence plays the role of the trigger in starting the next organized large scale deep convection in an atmosphere which is already preconditioned and prepared to sustain such deep convection.

The four time snapshots depicted in Fig. 6(A-D) are representative of the four different and important episodes of the large scale convective cycle. In Fig. 7, we plot the time series of the maximums of $\theta_{\mathrm{eb}}$ and $q$ (top), the heating maximums (middle), and the zonal mean of $\theta_{\mathrm{eb}}, q, \theta_{1}+$ $\gamma_{2} \theta_{2}$ (bottom). In the present parameter regime, where the convectively coupled waves are isolated such zonal means are a measure of the wave behavior itself. According to the discussion above the following four phases are highlighted in Fig. 7.
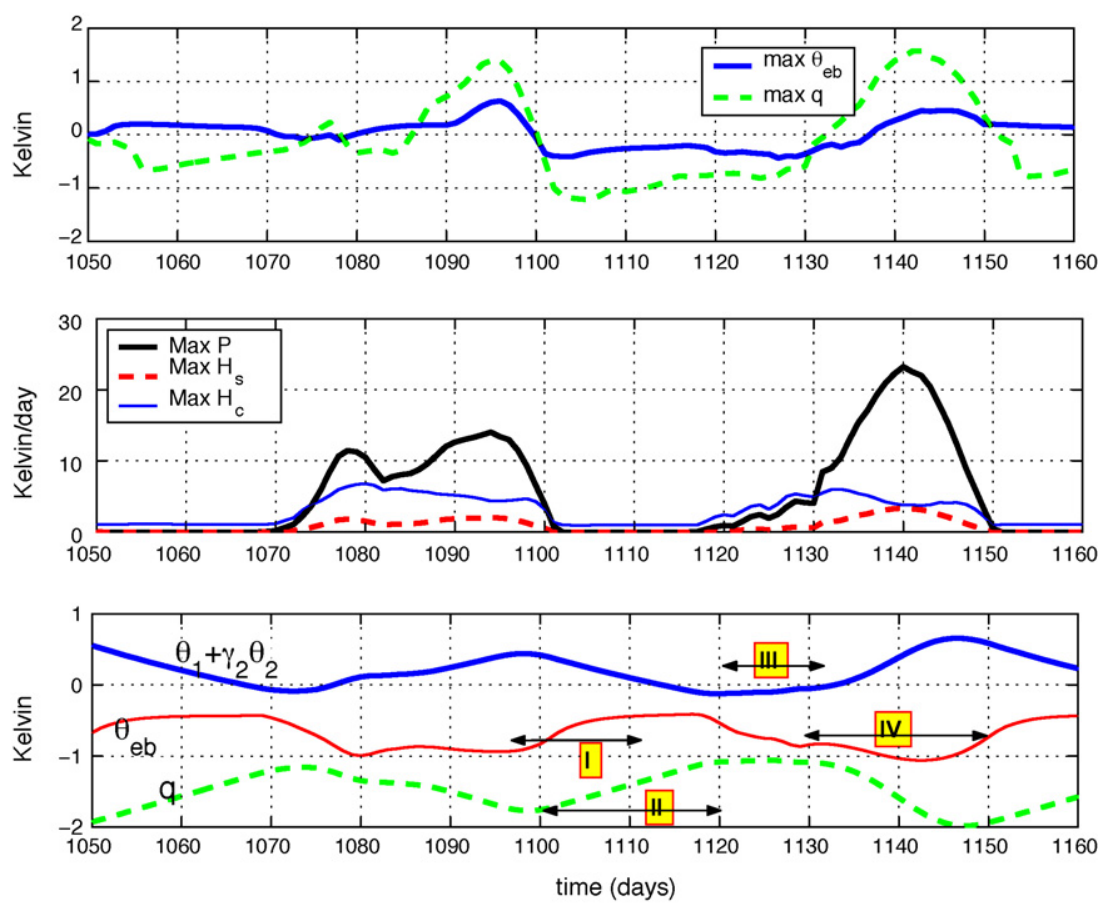

Fig. 7. The large scale convective cycle seen through the time series of the maximums of $\theta_{\mathrm{eb}}$ and $q$ (top), the heating rate maximums (middle): the congestus max is rescaled by a factor of 100, and the zonal means of $\theta_{\mathrm{eb}}, q, \theta_{1}+\gamma_{2} \theta_{2}$ (bottom). Four different phases are identified on the last panel: (I) regeneration of $\theta_{\mathrm{eb}}$, (II) preconditioning, (III) birth of convective activity and organization of moist waves, and (IV) amplification, CAPE consumption, drying, and decay of wave. 


\subsubsection{Regeneration phase}

The regeneration phase starts during the highest peak of the previous convective event and lasts about 12 days, which is comparable to the evaporative time scale of $\tau_{\mathrm{e}}=9$ days. The increase in $\theta_{1}+\gamma_{2} \theta_{2}$ anomalies from the previous convective event yields a weakening of the downward mass flux, $D$, by increasing $\theta_{\mathrm{em}}$ while $\theta_{\mathrm{eb}}$ is already weak, thus leaving room for the surface evaporation $E$ to restore $\theta_{\mathrm{eb}}$, thus CAPE.

\subsubsection{Preconditioning phase}

The preconditioning phase starts right after the demise of the previous convective event and lasts about 20 days. It is the longest phase and it overlaps the regeneration phase. It is associated with both the increase (restoration) of the moisture level by the "evaporation of shallow/congestus clouds" as discussed above through the downdraft mass flux forcing and the decrease of the combination $\theta_{1}+\gamma_{2} \theta_{2}$ via radiative cooling. The period of 20 days corresponds roughly to the time it takes for $\theta_{1}+\gamma_{2} \theta_{2}$ to cool down by about $0.8 \mathrm{~K}$ under the sole effect of the imposed radiative cooling of $Q_{R, 0}^{1}=0.04 \mathrm{~K} \mathrm{day}^{-1}$ which is comparable to the amount by which $\theta_{1}+\gamma_{2} \theta_{2}$ actually decreases on average during this period from the bottom panel of Fig. 7. Notice that the Newtonian relaxation time of $\tau_{\mathrm{D}}=50$ days cannot justify (alone) this decrease in $\theta_{1}+\gamma_{2} \theta_{2}$ during this relatively short period of time. Moreover, recall that in the absence of precipitation, $P=0, q$ increases from downdrafts at a rate roughly set by the RCE value, since the perturbations in $\theta_{\mathrm{eb}}-\theta_{\mathrm{em}}$ are relatively small,

$$
\frac{m_{0}}{H_{\mathrm{T}}}\left(1+\frac{\mu_{2}}{\bar{P}}\left(\bar{H}_{\mathrm{s}}-\bar{H}_{\mathrm{c}}\right)\right)^{+}\left(\bar{\theta}_{\mathrm{eb}}-\theta_{\mathrm{em}}\right)=\frac{h_{\mathrm{b}}}{H_{\mathrm{T}}} \frac{1}{\tau_{\mathrm{e}}}\left(\theta_{\mathrm{eb}}^{*}-\bar{\theta}_{\mathrm{eb}}\right) \approx 0.034 \mathrm{Kday}^{-1}
$$

leading to a moistening equivalent to about $0.7 \mathrm{~K}$ during the time period of 20 days, which is also comparable to the amount by which $q$ has raised during the preconditioning period. Notice that with the level of $\theta_{\mathrm{eb}}$ being already restored during the first phase, these last two effects contribute to the overall increase of $P_{0}$, which ultimately becomes positive and thus provides a potential for deep convective.

\subsubsection{Birth and organization phase}

When the middle troposphere is moist a fraction of the freshly generated positive part of $P_{0}$, during the previous preconditioning and regeneration episodes, is converted into actual heating and precipitation thanks to the decrease of the value of the switch parameter due to congestus moistening. This heating then excites first baroclinic waves which in turn induce fluid motion providing the necessary local increase for $q$ through moisture convergence to amplify the deep convection.

\subsubsection{The amplification, CAPE consumption, and decaying phase}

After the organization phase where the convectively coupled wave forms and starts to propagate, further and much stronger amplification of the convection is achieved as demonstrated by the middle panel in Fig. 7. This amplification is associated with the sole local effects induced by the wave activity since, at this time, the zonal means of $q$ and $\theta_{\mathrm{eb}}$ are not increasing and the zonal mean of $\theta_{1}+\gamma_{2} \theta_{2}$ is not decreasing, as seen on the bottom panel but the maxima in the top panel are indeed increasing. On the other hand, the relatively low values of $\theta_{1}+\gamma_{2} \theta_{2}$, seen during the beginning of the amplification stage, induce a substantial decrease in $\theta_{\mathrm{em}}$ compensating for the increase in $q$ thus maintaining a certain level of downdrafts which helps reduce the increase in $\theta_{\mathrm{eb}}$ thus limiting further CAPE build-up. In turn, the high deep-convective rates consume CAPE 
by both raising the value of $\theta_{1}+\gamma_{2} \theta_{2}$ and increasing the downdrafts, through the increase of stratiform heating, which in turn yield the decrease of $\theta_{\mathrm{eb}}$. Also, the precipitation provokes a sudden drop in moisture level which in turn tends to decrease the deep heating rate and forces the wave to decay. Moreover, the increased value of $\theta_{1}+\alpha_{2} \theta_{2}$ compensates for the negative effect of $q$ on $\theta_{\mathrm{em}}$, and thus weakens the downdrafts and prevents any immediate restoration of $q$ but also has a direct negative effect on $P_{0}$ through $a_{0}$, forcing it toward negative values, thus preventing immediate regeneration of deep convection.

The central role played by the combination $\theta_{1}+\gamma_{2} \theta_{2}$ during both the preconditioning and the demise phases of the large scale convective cycle clarifies why the parameter $a_{0}$ is so crucial for both the amplification and intermittency of the convective activity (cf. Fig. 2). As an inverse time scale factor, it has the important role of simultaneously competing with the convective heating, the radiative cooling, and the moistening time scales.

Also although the downdrafts seem to play an important role in restoring the moisture level during the preconditioning phase as discussed above, this effect alone cannot justify the abrupt local increase in $q$ in front of the convective region during the mature stage of wave in Fig. 6(B), by about $4 \mathrm{~K}$ during the short time period of $12,500 \mathrm{~km} /\left(18 \mathrm{~m} \mathrm{~s}^{-1}\right) \approx 8$ days, assuming that by the time the wave arrives at $x=10,000 \mathrm{~km}, q$ will rise from its 'current' value of about $-2.5 \mathrm{~K}$ to a comparable convective peak of about $1.5 \mathrm{~K}$. This, in essence, confirms the role played by the low level convergence in raising locally the moisture and hence providing the fuel for deep convection.

\section{Concluding discussion}

Here, we analyzed the detailed evolution of convectively coupled waves in a multi-cloud convective parametrization introduced recently by Khouider and Majda (2006a) and summarized in Section 2 when all the nonlinear switches of the convective parametrization are activated while both the rotation (beta effect) and the nonlinear advection are ignored. The regime of wave dynamics centered around a sounding associated with an RCE with the relatively weak radiative cooling, $Q_{R, 1}^{0}=0.04 \mathrm{Kday}^{-1}$, was utilized here to establish a regime of chaotic intermittent nonlinear dynamics involving the birth and demise on large scale nonlinear convectively coupled waves which are isolated events in space and time. This allows us to elucidate and isolate the variety of nonlinear mechanisms operating in the model parametrization which lead to large scale convective wave organization as described in Section 3.

In particular, as illustrated in Fig. 6(D), during the preconditioning stage, radiative cooling and regeneration of $\theta_{\mathrm{eb}}$ act to precondition the atmosphere to have high CAPE with a relatively dry upper troposphere; in this preconditioned stage, the lower troposphere is continuously moistened through the creation of congestus clouds so that the additional low level moisture convergence associated with second baroclinic gravity waves creates a locally enhanced moisture anomaly as shown clearly in Fig. 6(D). It has been shown in Section 3 that when such enhanced moisture anomalies are carried along with the dry gravity wave, and encounter a region of enhanced $\theta_{\mathrm{eb}}$, they trigger the birth stage of large scale deep convection as illustrated in Fig. 6(D).

We make two additional points about this process. First, it illustrates one crucial role of both congestus moistening and moisture convergence from second baroclinic gravity waves in the model parametrization which are novel features of the parametrization. Second, while the details of the nonlinear dynamics described here are completely different and involve second baroclinic gravity waves, the four stages of wave evolution in Section 3 are qualitatively similar to those occurring in the western warm pool after a dry intrusion (Parsons et al., 2000). It would be 
interesting to extend the present model parametrization to an equatorial beta plane to pursue this analogy in greater detail among other applications.

\section{Acknowledgments}

The research of B.K. is supported by a University of Victoria Start-up grant and a grant from the Natural Sciences and Engineering Research Council of Canada. The research of A.M. is partially supported by ONR N0014-96-1-0043, NSF \# DMS-0456713, and NSF-FRG DMS-0139918. The authors are thankful to M.W. Moncrieff for sponsoring a visit for B.K. to NCAR during the summer of 2005 where a part of this work was initiated.

\section{References}

Arakawa, A., Shubert, W.H., 1974. Interaction of a cumulus cloud ensemble with the large-scale environment. Part I. J. Atmos. Sci. 31, 674-701.

Biello, J., Majda, A.J., 2005. A multi-scale model for the Madden-Julian oscillation. J. Atmos. Sci. 62, 1694-1721.

Charney, J.G., Eliassen, A., 1964. On the growth of the hurricane depression. J. Atmos. Sci. 21, 68-75.

Dunkerton, T.J., Crum, F.X., 1995. Eastward propagating 2- to 15-day equatorial convection and its relation to the tropical intraseasonal oscillation. J. Geophys. Res. 100, 25781-25790.

Emanuel, K.A., 1987. An air-sea interaction model of intraseasonal oscillations in the tropics. J. Atmos. Sci. 44, 23243240.

Emanuel, K.A., Neelin, J.D., Bretherton, C.S., 1994. On large-scale circulations in convecting atmosphere. Quart. J. R. Meteor. Soc. 120, 1111-1143.

Frierson, D., Majda, A.J., Pauluis, O., 2004. Dynamics of precipitation fronts in the tropical atmosphere. Comm. Math. Sci. 2, 591-626.

Fuchs, Z., Raymond, D., 2002. Large-scale modes of a nonrotating atmosphere with water vapor and cloud-radiation feedbacks. J. Atmos. Sci. 59, 1669-1679.

Gill, A.E., 1982. Atmosphere Ocean-Dynamics. Academic Press, 666 pp.

Hayashi, Y., 1971. Large-scale equatorial waves destabilized by convective heating in the presence of surface friction. J. Meteor. Soc. Jpn. 49, 458-466.

Haertel, P.T., Kiladis, G.N., 2004. On the dynamics of two day equatorial disturbances. J. Atmos. Sci. 61, $2707-2721$.

Johnson, R.H., Rickenbach, T.M., Rutledge, S.A., Ciesielski, P.E., Schubert, W.H., 1999. Trimodal characteristics of tropical convection. J. Climate 12, 2397-2407.

Khouider, B., Majda, A.J., 2006a. A simple multicloud parametrization for convectively coupled tropical waves. Part I: Linear analysis. J. Atmos. Sci. 63, 1308-1323.

Khouider, B., Majda, A.J., 2006b. A simple multicloud parametrization for convectively coupled tropical waves. Part II: Nonlinear simulations. J. Atmos. Sci., in press.

Khouider, B., Majda, A.J., 2005a. A non-oscillatory well balanced scheme for an idealized tropical climate model. Part I: Algorithm and validation. Theor. Comput. Fluid Dyn. 19, 331-354.

Khouider, B., Majda, A.J., 2005b. A non-oscillatory well balanced scheme for an idealized tropical climate model. Part II: Nonlinear coupling and moisture effects. Theor. Comput. Fluid Dyn. 19, 355-375.

Kiladis, G.N., Straub, K., Haertel, P., 2005. Zonal and vertical structure of the Madden-Julian oscillation. J. Atmos. Sci. 62, 2790-2809.

Lindzen, R.S., 1974. Wave-CISK in the tropics. J. Atmos. Sci. 31, 156-179.

Lin, X., Johnson, R.H., 1996. Kinematic and thermodynamic characteristics of the flow over the Western Pacific Warm Pool during TOGA COARE. J. Atmos. Sci. 53, 695-715.

Majda, A.J., Biello, J., 2004. A multi-scale model for the intraseasonal oscillation. Proc. Natl. Acad. Sci. 101, 4736-4741.

Majda, A.J., Khouider, B., 2002. Stochastic and mesoscopic models for tropical convection. Proc. Natl. Acad. Sci. 99, 1123-1128.

Majda, A.J., Khouider, B., Kiladis, G.N., Straub, K.H., Shefter, M.G., 2004. A model for convectively coupled tropical waves: nonlinearity, rotation, and comparison with observations. J. Atmos. Sci. 61, 2188-2205.

Majda, A.J., Shefter, M., 2001a. Waves and instabilities for model tropical convective parameterizations. J. Atmos. Sci. $58,896-914$. 
Majda, A.J., Shefter, M., 2001b. Models for stratiform instability and convectively coupled waves. J. Atmos. Sci. 58, 1567-1584.

Mapes, B.E., 1993. Gregarious tropical convection. J. Atmos. Sci. 50, 2026-2037.

Mapes, B.E., 2000. Convective inhibition, subgridscale triggering energy, and "stratiform instability" in a toy tropical wave model. J. Atmos. Sci. 57, 1515-1535.

Moncrieff, M.W., Klinker, E., 1997. Organized convective systems in the tropical western Pacific as a process in general circulation models: a TOGA-COARE case study. Quart. J. R. Meteor. Soc. 123, 805-827.

Nakazawa, T., 1988. Tropical super clusters within intraseasonal variations over the western Pacific. J. Meteor. Soc. Jpn. 66, 823-839.

Neelin, J.D., Yu, J., 1994. Modes of tropical variability under convective adjustment and Madden-Julian oscillation. Part I: Analytical theory. J. Atmos. Sci. 51, 1876-1894.

Parsons, D.B., Yoneyama, K., Redelsperger, J.L., 2000. The evolution of the tropical western Pacific atmosphere-ocean system following the arrival of a dry intrusion. Quart. J. R. Meteor. Soc. 126, 517-548.

Slingo, J.M., Sperber, K.R., Boyle, J.S., Ceron, J.-P., Dix, M., Dugas, B., et al., 1996. Intraseasonal oscillation in 15 atmospheric general circulation models: results from an AMIP diagnostic subproject. Climate Dyn. 12, 325-357.

Straub, K.H., Kiladis, G.N., 2002. Observations of a convectively-coupled Kelvin wave in the eastern Pacific ITCZ. J. Atmos. Sci. 59, 30-53.

Wheeler, M., Kiladis, G.N., 1999. Convectively coupled equatorial waves: analysis of clouds and temperature in the wavenumber-frequency domain. J. Atmos. Sci. 56, 374-399.

Wheeler, M., Kiladis, G.N., Webster, P.J., 2000. Large scale dynamical fields associated with convectively coupled equatorial waves. J. Atmos. Sci. 57, 613-640.

Yamasaki, M., 1969. Large-scale disturbances in a conditionally unstable atmosphere in low latitudes. Pap. Meteor. Geophys. 20, 289-336.

Yano, J.-I., Emanuel, K.A., 1991. An improved model of the equatorial troposphere and its coupling to the stratosphere. J. Atmos. Sci. 48, 377-389.

Yano, J.-I., McWilliams, J.C., Moncrieff, M.W., Emanuel, K.A., 1995. Hierarchical tropical cloud systems in an analog shallow-water model. J. Atmos. Sci. 52, 1723-1742.

Yano, J.-I., Moncrieff, M.W., McWilliams, J.C., 1998. Linear stability and single column analyses of several cumulus parametrization categories in a shallow-water model. Quart. J. R. Meteor. Soc. 124, 983-1005.

Zehnder, J.A., 2001. A comparison of convergence- and surface-flux-based convective parameterizations with applications to tropical cyclogenesis. J. Atmos. Sci. 58, 283-301. 